\title{
Systematic Revision of the Genus Pancratium L. (Amaryllidaceae) in Egypt with a New Addition
}

\author{
Azza EL-HADIDY, Monier ABD EL-GHANI, Wafaa AMER, Rania HASSAN \\ Cairo University, Faculty of Science, The Herbarium, Giza 12613, Egypt; elghani@yahoo.com (corresponding author)
}

\begin{abstract}
In Egypt, taxonomic aspects of the genus Pancratium L. (Amaryllidaceae, Pancratieae) were thoroughly studied to point out the most reliable characters for taxon delimitation. Here, we base our species on both geographical and morphological features. The detailed taxonomic revision of genus Pancratium is based on field studies and the examination of representative specimens together with the authentic types deposited in the major herbaria of Egypt and Kew. Variations in the aerial scape, spathe, perianth, corona-teeth, pistil, stamens, capsules and seeds, as well as those of leaf base and leaf orientation are good markers to separate the different species. As a result, five species are recognized, including $P$. trianthum as a new record to the flora of Egypt. It is restricted to the south-eastern corner of Egypt (Gebel Elba region) and being unique in possessing solitary spathe and long attenuate leaf base. A specific key and descriptions are given with typifications.
\end{abstract}

Keywords: Egyptian flora, new records, Pancratieae, Pancratium trianthum, taxonomy

\section{Introduction}

Amaryllidaceae is well-marked by its showy lily-like epigynous flowers as in common onion. According to Meerow and Snijman (1998), Amaryllidaceae is about 800 species in 59 genera. Its center of diversity is in South America (28 genera) and Africa (19 genera). Eight genera are growing in Mediterranean and temperate regions of Asia, while only Crinum L., is represented in both the Old and New Worlds. It forms one of the climax groups in order Asparagales (Dahlgren et al., 1985; Fay and Chase, 1996). They are probably more closely related to the $\mathrm{Al}$ liaceae (formerly Liliaceae) and Hyacinthaceae but not to Hypoxidaceae, Agavaceae, Haemodoraceae or Alstroemeriaceae, with which they have formerly been united. It is separated from Liliaceae by its inferior ovary, in addition to its umbellate inflorescence with the characteristic bracts (spathe); the only character formerly separating the two families (Hutchinson, 1959). Species belonged to this family are mostly bulbous rarely rhizomatous herbaceous perennials of diverse habitats.

Many species represented as endangered species like Pancratium maritimum in its original range, the sandy coasts of the Mediterranean displaying the Sea (Grassi et al., 2005). Other species like Pancratium sickenbergeri is subjected to high levels of grazing. It is commonly found on sand dunes of the Negev Desert (Israel), and characterized by three phenological stages consisting, respectively, of inflorescence, leaves and no above-ground biomass: all stages are subject to herbivory by dorcas gazelles (Ward and Saltz, 1994; Saltz and Ward, 2000). When there is no aboveground biomass, the gazelles dig for underground parts of $P$. sickenbergeri and may consume all or part of the bulb, which contains most of the plant's volume (Ward and Saltz, 1994).

Within the framework of Engler and Prantl Pax (1887) and Pax and Hoffmann (1930) gave a comprehensive revision of the family Amaryllidaceae. According to the habit of the plant and the type of the inflorescence, they reclassified the Amaryllidaceae into 4 subfamilies: Amaryllidoideae, Agavoideae, Hypoxidoideae and Campanematoideae. Furthermore, they used the presence or absence of corona to subdivide Amaryllidoideae into 2 tribes: Amaryllideae and Narcisseae. Moreover, the former is divided into 6 subtribes; while the latter into 5 tribes. They included the genus Pancratium within the subtribe Eucharidinae of the tribe Narcissae. Hutchinson's classification (Hutchinson, 1934, 1959) was the first crucial recircumscription of Amaryllidaceae, defining the unifying character of the family to be an umbellate inflorescence subtended by an involucre of one or more spatheceous bracts. He used both vegetative and floral characters to classify the family into 13 tribes and included Pancratium within the tribe Eucharideae. Now, the present generic circumscription of the genus Pancratium within the tribe Pancratieae of Amaryllidaceae s. str., has gained wide acceptance (Dahlgren et al., 1985; Meerow, 1995; Meerow and Snijman, 1998; Müller-Doblis and Müller-Doblis, 1996; Traub, 1963). This is due to the presence of conspicuous false corona formed by the basal connation of the staminal filaments.

This treatment was accepted by many authors. The works of Andrews (1956), Baker (1898), Björnstad (1973), Dinsmore (1933), El-Gadi (1978), Feinbrun-Dothan (1986), Heller and Heyn (1991), Maire (1959), Meikle (1985), Mill (1984), and Morton (1965) are among the 
most useful contributions of Pancratium, especially for Egypt region.

In Egypt, Amaryllidaceae is represented by two genera Pancratium and Narcissus, the latter is more widespread and common than the former. Therefore, this study focuses on the genus Pancratium as little attention has been paid to its taxonomy. Forsskal (1775) and Delile (1813-1814) reported the occurrence of one species, whereas Boissier (1882) added two other species, of which $P$. tortifolium has been newly described. Sickenberger (1901) added another new species; $P$. arabicum. Altogether, four species of Pancratium (viz., Pancratium maritimum L., P. tortuosum Herb., P. sickenbergeri Asch. and Schweinf. ex Barb.Boiss. and Barbey, and P. arabicum Sickenb.) are reported by Boulos (2005), ), El Hadidi and Fayed (1994/1995), Täckholm (1974) and Täckholm and Drar (1954). Pancratium aegyptiacum is conspecific to $P$. arabicum on the basis of the floral characters (especially the perianth). This study included several characters that are not used by Boulos (2005) in d delimiting its species. Moreover, synonyms are improved and some others are also included. Our proposed key is not dependent on Boulos's work (2005), as it included more morphological diagnostic characters. The aim of this study is to fill the gap in our knowledge of the taxonomy of the genus Pancratium in Egypt, by providing a critical revision of its species, clarifying some older and neglected ones, studying in more detail the most reliable taxonomic characters for the identification of taxa, with similar habitats. This revision revealed the presence of five species, of which $P$. trianthum Herb. is a new addition to the Flora of Egypt.

\section{Material and methods}

Morphological data were scored from examinations of Egyptian herbarium specimens as well as the authentic ones kept in Kew $(\mathrm{K})$; examination of fresh material during field trips; and the contribution sources of Björnstad (1973), Boulos (2005), Feinbrun-Dothan (1986), Morton (1965), Täckholm (1974) and Täckholm and Drar (1954).

Herbarium study is based on the examination over 500 herbarium specimens kept in the different Egyptian major herbaria as well as the authentic specimens related to the studied Pancratium species. These herbaria were annotated during this study as Cairo University (CAI), the Agricultural Museum (CAIM), the National Research Centre (CAIRC) and the Royal Botanic Gardens in England (K) (acronyms follow Holmgren et al., 1998).

Field work was conducted over 4-year period (20022006) to obtain fresh material in their natural habitats for the in vivo study of the floral characters, for preparing exsiccate, as well as to gather eco-geographical data, and field observations were made in several localities. (Tab.1). The examined representative specimens were geographically arranged according to the phytogeographical territories of
Egypt proposed by El Hadidi (2000; 14-22). Local geographic distributions have been plotted exclusively using the information from herbarium sheets and field trips. Habitat, flowering period, and common names were provided using the available data on herbarium specimen labels and those reported in the protologue of each taxon. In this study, the species were arranged chronologically, and abbreviations of the authors' names followed Brummitt and Powel (1992). Type and authentic specimens for each taxon were seen by the authors and are followed by (!).

Tab. 1. Locations of the collected Pancratium species

\begin{tabular}{|c|c|c|c|}
\hline Longitudes & Latitudes & Locality & Species \\
\hline $29^{\circ} 54^{\prime}$ & $30^{\circ} 89^{\prime}$ & Burg El Arab & \multirow{5}{*}{$\begin{array}{l}\text { Pancratium } \\
\text { maritimum L. }\end{array}$} \\
\hline $27^{\circ} 85^{\prime}$ & $31^{\circ} 23^{\prime}$ & Ras El Hekma & \\
\hline $27^{\circ} 24^{\prime}$ & $31^{\circ} 34^{\prime}$ & Mersa Matruh & \\
\hline $25^{\circ} 91^{\prime}$ & $31^{\circ} 60^{\prime}$ & Sidi Barani & \\
\hline $25^{\circ} 16^{\prime}$ & $31^{\circ} 56^{\prime}$ & Sallum & \\
\hline $33^{\circ} 01^{\prime}$ & $31^{\circ} 03^{\prime}$ & Bir El Abd & $\begin{array}{l}\text { P. sickenbergeri Asch. } \\
\text { and Schweinf. ex Barb.- } \\
\text { Boiss. and Barbey }\end{array}$ \\
\hline $33^{\circ} 58^{\prime}$ & $31^{\circ} 06^{\prime}$ & El Arish & \multirow{3}{*}{ P. arabicum Sickenb. } \\
\hline $34^{\circ} 09^{\prime}$ & $31^{\circ} 20^{\prime}$ & $\begin{array}{c}\text { Sheikh } \\
\text { Zuwayied }\end{array}$ & \\
\hline $34^{\circ} 16^{\prime}$ & $31^{\circ} 27^{\prime}$ & Rafah & \\
\hline $35^{\circ} 94^{\prime}$ & $22^{\circ} 06^{\prime}$ & $\begin{array}{c}\text { Wadi Yahameib } \\
\text { (Gebel Elba) }\end{array}$ & P. tortuosum Herb. \\
\hline
\end{tabular}

Results

Pancratium L.

Sp. Pl., ed.1, 290 (1753)-Gen. Pl., ed. 5, 141 (1754).

\section{Synonyms}

Zouchia Raf., Fl. Tellur. 4: 22 (1838).

Halmyra Salisb. ex Parl., Nuovi Gen. Sp. Monocot.: 28 (1854).

Bollaea Parl., Bull. Soc. Bot. Fr. 5 (1): 509 (1858); [non Bollea Rchb. f., 1852].

Almyra Salisb., Gen. Pl. 108 (1866).

Chapmanolirion Dinter, Deutsch Sudw.-Afr. Fragm.: 49 (1909).

\section{Type species: Pancratium zeylanicum L.}

A palaeotropical genus with 16 species (Mabberley 1993) distributed from Macaronesia, Mediterranean basin and throughout Africa to Tropical Asia, also introduced and cultivated in many countries.

\section{Key to the species of Pancratium}

1. Perianth $16-22 \mathrm{~cm}$ long; perianth-tube $11-16 \mathrm{~cm}$ long, 2-3 times as long as the perianth-segments; coronateeth acuminate; style glaucous, $16-22 \mathrm{~cm}$ long; aerial scape dilated at the base; plants confined to Gebel Elba region. 
26

1'. Perianth 6-16 cm long; perianth-tube $2-12 \mathrm{~cm}$ long, shorter than or up to 2 times as long as the perianthsegments; corona-teeth acute; style white, $6-16 \mathrm{~cm}$ long; aerial scape dilated at the tip; plants of Mediterranean and desert regions.

2. Spathe 1-valved, $2-4 \mathrm{~cm}$ diam., apex bifid, margins contorted at the base; corona-teeth 7-11 mm long; free part of filaments 10-15(-20) mm long; leaves slightly twisted developing with or after flowers, base long attenuate, sheath absent. 3. P. trianthum

2' Spathe 2-valved, 1-2 cm diam., apex entire, margins imbricate; corona-teeth $5-7 \mathrm{~mm}$ long; free part of filaments 6-10 mm long; leaves spirally twisted developing with the flowers, base truncate, sheath present... 2. P. tortuosum

3. Flowers 2-5(-6), $7-10 \mathrm{~cm}$ long; perianth-tube $2-4 \mathrm{~cm}$ long, shorter than the perianth-segments or rarely equal; free part of filament 3-5 mm long; ovary 1-2 cm long, 2-3 times as long as broad; spathe $3-5 \mathrm{~cm}$ long; aerial scape 0.3 $0.5 \mathrm{~cm}$ diam. at the tip; leaves decumbent on the soil surface, $10-30 \times 0.3-1.0 \mathrm{~cm}$, broadest at the middle or below, conspicuously spirally twisted along its whole length; seeds 6-9 × $3-7 \mathrm{~mm}$ 4. P. sickenbergeri

3'. Flowers 10-18 cm long; perianth-tube $5-12 \mathrm{~cm}$ long, longer than the perianth-segments; free part of filament $5-10 \mathrm{~mm}$ long; ovary $2-3 \mathrm{~cm}$ long, at least 3 times as long as broad; spathe 5-8 cm long; aerial scape 0.7-3.0 $\mathrm{cm}$ diam. at the tip; leaves erecto-patent above the soil surface, $15-80 \times 1.0-3.0 \mathrm{~cm}$, broadest at the middle or above, straight or arched to slightly twisted near the apex; seeds 10-14 × 6-10 mm

4. Perianth-tube 5-8 cm long, perianth-segments 3-5 $\mathrm{cm}$ long, with narrow green mid-stripe (2-4 $\mathrm{mm}$ diam.), corona 2-3 cm long; anthers 5-10 mm long; fruit 2-3 x 1.5$2.5 \mathrm{~cm}$; leaves without scarious-membranous sheath at the base.

1. P. maritimum

4 . Perianth-tube 8-12 cm long; perianth-segments 5-7 cm long, with broad green mid-stripe (4-6 $\mathrm{mm}$ diam.); corona (2.5-)3-4 cm long; anthers 10-17 mm long; fruit $3-5 \times 2.5-3.5 \mathrm{~cm}$; leaves with scarious-membranous sheath at the base. ...5. P. arabicum

1. Pancratium maritimum L. Sp. Pl., ed. 1, 291 (1753); Boiss., Fl. Orient. 5(1): 152 (1882)-Ibid. 5 (2): 756 (1884); Asch. and Schweinf., Ill. Fl. Egypte, Mém. Inst. Égypt. 2: 149 no. 1031 (1887)-Ibid., Suppl.: 774 (1889); Asch. in Asch. and Scweinf., Ibid., Suppl.: 805 no. 242, 813 no. 48 (1889); Sickenb., Contrib. Fl. Égypte, Mém. Inst. Egypt. 4 (2): 289 (1901); Muschl., Man. Fl. Egypt 1: 235 (1912); Ramis, Tabell. Fl. Aegypt.: 59 (1929); Dinsm. in Post, Fl. Syr., Pal. et Sn., ed. 2, 2: 609 (1933); Blatt., Fl. Arab. 456 (1939); O. Schwartz, Fl. Trop. Arab. 10: 354 (1939); Täckh. and Drar, Fl. Egypt 3: 359 (1954) [excl. P. aegyptiacum as a synonym]; Täckh., Stud.
Fl. Egypt, ed. 1, 457 (1956); Maire, Fl. Afr. Nord 6: 35 (1959); Quézel and Santa, Nouv. Fl. Alg. 1: 215 (1962); Mouterde, Nouv. Fl. Syrie 1: 292 (1966); Täckh., Stud. Fl. Egypt, ed. 2, 657 (1974); El-Gadi in Jafri and El-Gadi, Fl. Libya 51: 3 (1978); R. R. Mill. in Davis, Fl. Turkey 8: 380 (1984); Meikle, Fl. Cyprus 2: 1577 (1985); Danin et al., Willdenowia 15 (1): 308 (1985); Feinbrun in Zohary, Fl. Palaest. 4: 108 (1986); El Husseini and Hosni, Taeckholmia 12: 83 (1989); D. Heller and Heyn, Consp. Fl. Orient. 6: 53 (1991); El Hadidi and Fayed, Taeckholmia 15: 189 (1995); El Hadidi, Fl. Aegyptiaca 1(1): 32 (2000); Boulos, Fl. Egypt 4: 87 (2005).

Type specimen : The illustration (t. 10 f. 28) of "Narcissus maritimus" in Morison, Pl. Hist. Univ. 2: 365 (1680) [lectotype by Wijnands, for typification see Jarvis (2007)].

\section{Synonyms}

Pancratium illyricum Forssk., Fl. Aegypt.-Arab. 209 no. 36 (1775). [non L., 1753].

Type specimen: Egypt: Alexandria garden "Alexandriae, hortensis", 1761-62; Forssk. 1542, 1543 (C) [see Hepper and Friis (1994)].

Pancratium carolinianum L., Sp. Pl. ed. 1: 291 (1753).

Type specimen: Carolina [Lectotype by Smith and Garland, 2003; Jarvis, 2007].

Glabrous herbaceous perennial, $40-70 \mathrm{~cm}$ high; bulb solitary or gregarious, usually deeply buried, globose or subglobose to ovoid or oblong-ovoid, $5-10 \mathrm{~cm}$ long, 4-9 $\mathrm{cm}$ diameter narrowed above into a long cylindrical neck; neck (6-) 10-30 cm long; tunic brownish. Leaves (3-)512 , developing after flowers, glaucous, broadly linear to oblong-lanceolate or lorate, $15-50 \mathrm{~cm}$ long, $1-2 \mathrm{~cm}$ diameter, broadest at the middle or slightly above, erect, often twisted near the apex, abruptly narrowed above to a blunt or subacute apex, glabrous near the base; base truncate, without scarious membranous sheath. Aerial scape 1(-2), solid, compressed, glaucous, $10-30 \mathrm{~cm}$ long, dilated at the tip (1-2 cm diameter), glabrous. Spathe 2-valved, unequal, imbricate, lanceolate or ovate-lanceolate to subulate, 5-8 $\mathrm{cm}$ long, $1-2 \mathrm{~cm}$ diameter, gradually or abrubtly tapering above into an acute apex. Flowers (3-)5-10(-14), mediumsized, 10-15 cm long, 2-3 times as long as the spathe, fragrant, subsessile or shortly pedicelled; pedicels stout, 0.5 $1.0(-1.3) \mathrm{cm}$, shorter than the ovary; perianth white, $8-13$ $\mathrm{cm}$ long; perianth-tube 5-8 cm long, 1.5-2 times as long as the perianth-segments; perianth-segments white, with a narrow green mid-stripe outside ( $2-4 \mathrm{~mm}$ diameter), erecto-patent, $3-5 \mathrm{~cm}$ long, 3-10 $\mathrm{mm}$ diameter, outer slightly longer and broader, narrowly spathulate to lanceolateoblong, obtuse to subacute at the mucronate or shortly cuspidate apex; inner lanceolate to oblong-lanceolate, acute or subacute, perianth-segments 1.5-2 times as long 

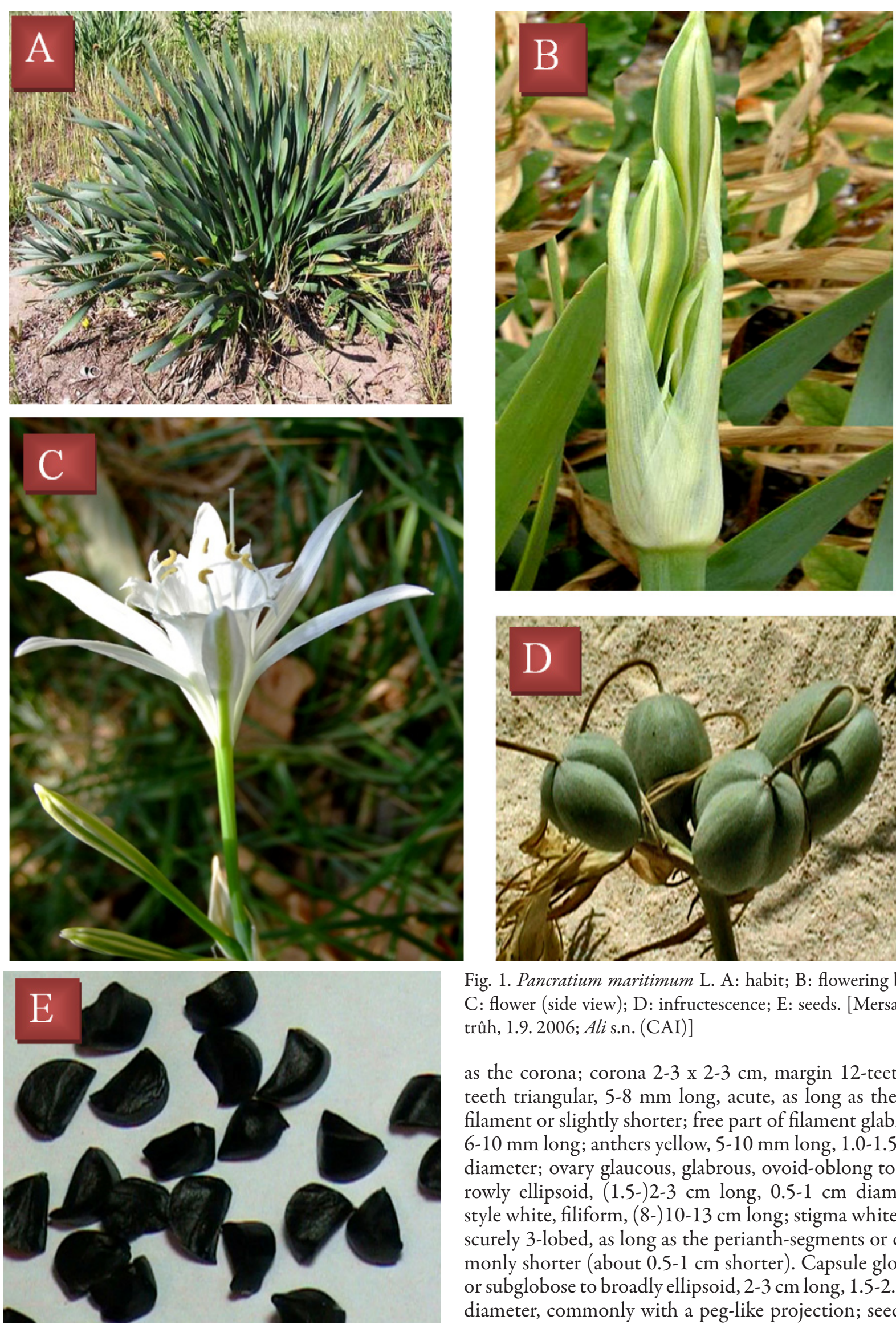

Fig. 1. Pancratium maritimum L. A: habit; B: flowering buds; C: flower (side view); D: infructescence; E: seeds. [Mersa Matrûh, 1.9. 2006; Ali s.n. (CAI)]

as the corona; corona $2-3 \times 2-3 \mathrm{~cm}$, margin 12-teethed; teeth triangular, $5-8 \mathrm{~mm}$ long, acute, as long as the free filament or slightly shorter; free part of filament glabrous, 6-10 mm long; anthers yellow, $5-10 \mathrm{~mm}$ long, $1.0-1.5 \mathrm{~mm}$ diameter; ovary glaucous, glabrous, ovoid-oblong to narrowly ellipsoid, (1.5-)2-3 cm long, $0.5-1 \mathrm{~cm}$ diameter; style white, filiform, (8-)10-13 cm long; stigma white, obscurely 3-lobed, as long as the perianth-segments or commonly shorter (about $0.5-1 \mathrm{~cm}$ shorter). Capsule globose or subglobose to broadly ellipsoid, $2-3 \mathrm{~cm}$ long, $1.5-2.5 \mathrm{~cm}$ diameter, commonly with a peg-like projection; seeds irregularly angular and compressed or cone-shaped, 10-13 
28

mm long, 6-10 mm diameter; testa black, sublustrous, minutely and closely foveolate (Fig. 1).

Representative specimens examined: Western Mediterranean coast: Sallum, 24.5.1963; V. Täckholm et al. s.n. (CAI)-Baltim, Mouth of Central Gharbiya drain, 26.6.1927; Simpson 5088 (CAIM)-Baltim, 19.4.1933; Sabet s.n. (CAI)-Baltim, sandy soil near the sea, 30.7.1938; Shabetai and Khattab s.n. (CAIM)-Baltim, on sandy hills, 29.8.1968; El Hadidi s.n. (CAI)-Sidi Barani, 1.9. 2006; Ali s.n. (CAI)-Mersa Matrûh, sandy soil, 17.6.1936; Shabetai 6044 (CAIM)-Mersa Matrûh, the peninsula between the harbour and the sea, 5.8.1953; Abd El Fadeel s.n. (CAI)-Mersa Matrûh, the peninsula between the harbour and the sea, 5.8.1953; Boulos s.n. (CAI)-Mersa Matrûh, 15.7.1959; Ghabbour s.n. (CAI)-Mersa Matrûh, 1.9. 2006; Ali s.n. (CAI)-Alexandria: Sidi Bishr to Mandara, abundant on the dunes in some places, 17.3.1923; Simpson 2065 (CAIM)-Alexandria: near Mandara, growing in clumps insands, 9.10.1923; Simpson 2186 (CAIM)Alexandria: Mandara, 13.8.1929; Hassib s.n. (CAI)-Ras El Hekma, 7.10.1963; Boulos and Kamal s.n. (CAI)-Ras El Hekma, 16.10.1963; Boulos s.n. (CAI)-Ras El Hekma, 18.10.1963; Boulos s.n. (CAI)-Ras El Hekma, 2.6.1964; $V$. Täckholm et al. s.n. (CAI)-Ras El Hekma, 2.9.2006; Ali s.n. (CAI)-Auf den Dünen von el Dachele, El Dikheila" westlich von Alexandrien, 7.10.1907; Hartmann s.n. (CAI)-El Dikheila, 14.7.1935; Shabetai z 5490 (CAIM)Mariut, N of Amirîya station, in the calcarious fields, 25.3.1927; G. Täckholm s.n. (CAI)-Mariut, 7.9.1968; Zabran and Girgis s.n. (CAI)-Mariut, 8.10.1968; Zahran and Girgis s.n. (CAI)-Mariut-Abu Sir, 8.4.1955; V. Täckbolm et al. s.n. (CAI)-Mariut-Abu Sir, Spring 1961; $V$. Täckholm s.n. (CAI)-Mariut-Burg El Arab, 9.3.1929; Hefnawy s.n. (CAI)-Mariut-Burg El Arab, 3.1930; Hassib s.n.
(CAI)-Near Abu Sir, 13.9.1908; Maire s.n. (CAI)-Abu Sir, 20.3.1953; Kamel s.n. (CAI)-Abu Sir, 20.3.1953; $V$. Täckholm s.n. (CAI)-Abu Sir, in the maritime dunes, 23.3.1961; $V$. Täckbolm et al. s.n. (CAI)-Abu Sir near Burg El Arab, 8.4.1955; Bot. Dept. Excr. s.n. (CAI)-Abu Sir, near Burg El Arab, in the moving dunes, 28.8.1957; $V$. Täckholm et al. s.n. (CAI)-Abu Sir near Burg El Arab, 27.5.1958; Boulos s.n. (CAI)-Abu Sir-Burg El Arab, beside the coast, 26.9.1971; Abbas and Khattab s.n. (CAIM)Burg El Arab, 2.9.2006; Ali s.n. (CAI)-Burg El Arab, 20.10.1976; El Khanagry s.n. (CAIM)-Um Sigheiw, sandy calcareous soil, 20.10.1976; El Khanagry s.n. (CAIM)Mariut, $5 \mathrm{~km}$ W Burg El Arab, Gharbaniat, sand dunes, 8.3.1979; Boulos s.n. (CAIRC).

Habitat: Sandy maritime seashores, sand-dunes, hill slopes and sometimes in calcareous fields. Flowering: JulySeptember.

Distribution: Egypt: All along the Mediterranean coastal region and Sinai (Fig. 2).

It occurs in Southern Europe (W, E) eastwards to W Transcaucasus, N Africa (Morocco to Egypt), Western Asia (Cyprus, Lebanon, Syria, Palestine, Sinai, Turkey) and Southeastern U.S.A. (Carolina).

2. Pancratium tortuosum Herb., Ann. Nat. Hist., ser. 1, 4: 28 (1840); M. Roem., Fam. nat. syn. monogr. 4: 178 (1847); Kunth, Enum. Pl. 5: 663 (1850); Baker, Handb. Amaryll. 120 (1888); Baker in Dyer (Ed.), Fl. Trop. Afr. 7: 406 (1898); Blatt., Fl. Ad. 339 (1916); Blatt., Fl. Arab. 456 (1936); O. Schwartz, Fl. Trop. Arab. 354 (1939); Täckh. and Drar, Fl. Egypt 3: 359 (1954); Andrews, Flow. Pl. Sudan 3: 289 (1956); Täckh., Stud. Fl. Egypt, ed. 1, 457 (1956); Täckh., Ibid., ed. 2, 657 (1974); Collonette, Ill. Flow. Saudi Arabia: 46 (1985); Migahid, Fl. SA, ed.

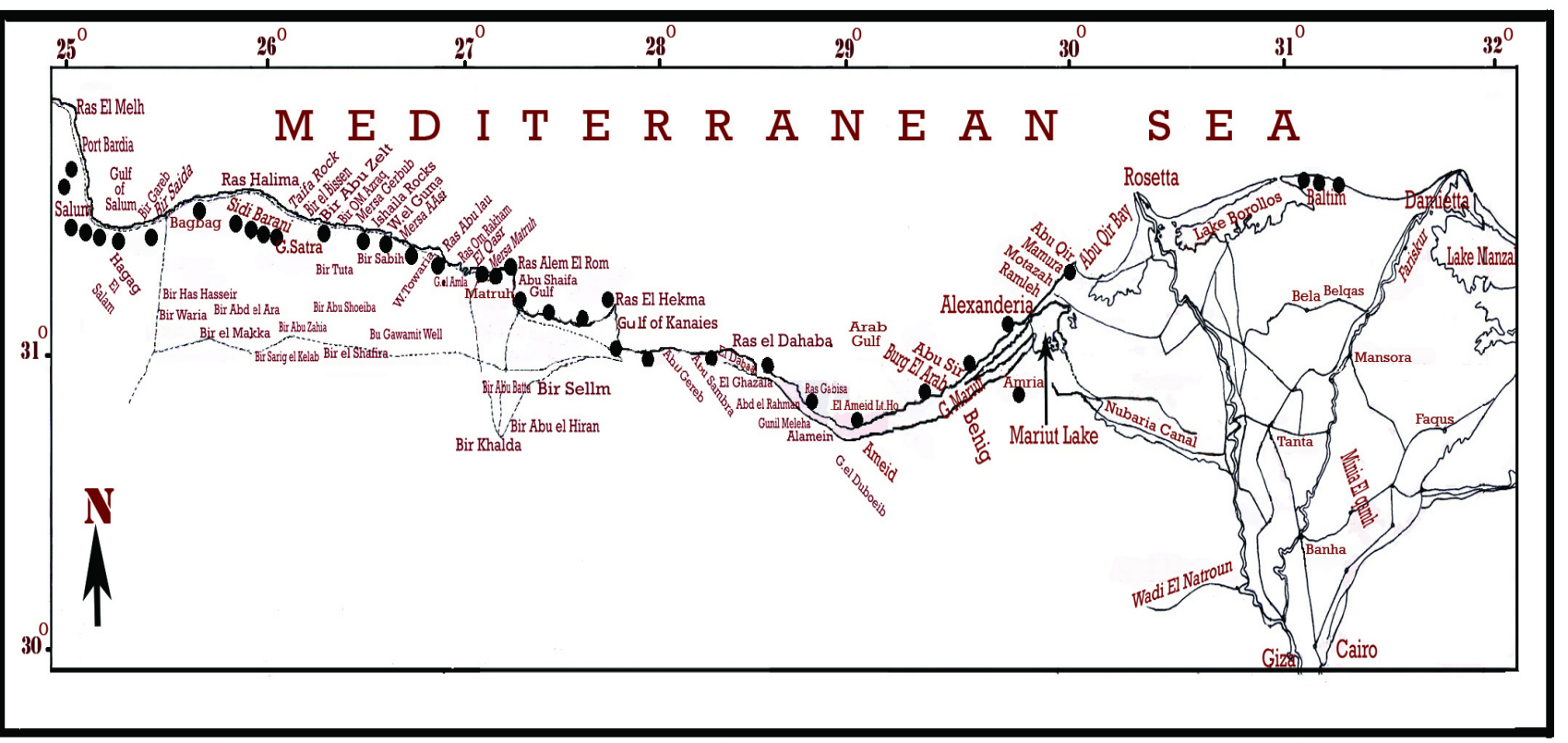

Fig. 2. Distribution mao of Pancratium maritimum; $\bullet$ specimens seen by the author 
3, 3: 13 (1989); D. Heller and Heyn, Consp. Fl. Orient. 6: 53 (1991); El Hadidi and Fayed, Taeckholmia 15: 189 (1995); Collonette, Wildflow. Saudi Arabia 40 (1999); El Hadidi, Fl. Aegyptiaca 1(1): 74 (2000); Boulos, Fl. Egypt 4: 87 (2005).

\section{Synonyms}

Pancratium tortifolium Boiss., Diagn. Pl. Orient., ser. 1, 13: 18 (1854).

Type specimen: Described from Arabia (Hedjaz): "In planitie deserti prope Dscheddam”, 20.12.1835; Schimper 876 (K, syntype !)-Arabia, 1837; Fischer 94 (K, syntype !).

Glabrous herbaceous perennial, 40-60 cm high; bulb solitary or gregarious, globose or subglobose to ovoid, 3-9 cm long, 3-7 cm diameter; neck (8-)10-21 cm long; tunic dark or blackish-brown. Leaves (3-)5-10, developing with the flowers, linear to lanceolate-linear or lorate, $10-40 \mathrm{~cm}$ long, $0.5-1.0 \mathrm{~cm}$ diameter, broadest at middle or below, erect, conspicuously spirally twisted, gradually tapering above into an acute apex, glabrous near the base; base truncate, with scarious-membranous sheath. Aerial scape 1(-2), solid, compressed, glaucous, $5-15(-20) \mathrm{cm}$ long, dilated at the glabrous base $(0.4-1.0 \mathrm{~cm}$ diameter). Spathe 2-valved, unequal, imbricate, elongated ovoid or lanceolate-subulate, (4.5-)5-8 cm long, 1-2 cm diameter, gradually tapering above into an acute apex. Flowers 2-5, large-sized, (18-)20-24 cm long, 3-4 times as long as the spathe, fragrant, pedicelled; pedicels stout, $(0.5-) 1-1.5 \mathrm{~cm}$, commonly shorter than the ovary, rarely equal; perianth white, (16-)18-22 cm long; perianth-tube 11-16 cm long, 2-3 times as long as the perianth-segments; perianth-segments white, with a green mid-stripe outside, patent, 5-7 $\mathrm{cm}$ long, outer narrowly spathulate-oblong or lanceolateoblong, gradually narrowed above to a mucronate apex; inner slightly shorter and narrower, lanceolate-linear, acute, $1.5-2$ times as long as the corona; corona $2-4 \times 2-3 \mathrm{~cm}$, margin 12-teethed; teeth triangular-acuminate or subulate, $5-8 \mathrm{~mm}$ long, as long as the free filament or shorter; free part of filament glabrous, 6-10(-12) mm long; anthers yellow, $5-10 \mathrm{~mm}$ long, 1.0-1.5 mm diameter; ovary glaucous, glabrous, $2-3 \mathrm{~cm}$ long, $1.0-1.5 \mathrm{~cm}$ diameter; style glaucous, filiform, $16-20 \mathrm{~cm}$ long; stigma obscurely 3-lobed, subequal to the perianth-segments or commonly shorter (about $0.5-0.7 \mathrm{~cm}$ shorter). Capsule oblong-ellipsoid, $2.5-3.5 \mathrm{~cm}$ long, $1.5-2 \mathrm{~cm}$ diameter, tapering above to an inconspicuous neck; seeds irregularly angular, $4-6 \mathrm{~mm}$ long, 3-5 mm diameter, testa black, sublastrous, minutely foveolate (Fig. 3).

Representative specimens examined: Arabian Desert: Wadi Dagalâi, 4.2.1933; Shabetai z 2333 (CAIM)Wadi Hodein, Dif and Abraq, in desert sand, 5.4.1943; Negumi Pacha 4726 (CAIM)-Wadi Selilo, on the hills of Hamra and the adjacent wadies, 7.2.1932; Drar s.n. (CAIM)-Upstream of Wadi Di-ib, affluent of Wadi
Kiraf, 60-70 km W of G. Elba, 30.1.1979; Boulos 12846 (CAIRC). Red Sea coast: Exp. to G. Elba district: Coastal plain between Abu Ramad and Mersa Na'am, 5.2.1962; V. Täckholm et al. s.n. (CAI)-Mersa Halâib, 22.1.1929; G. Täckholm s.n. (CAI). Gebel Elba: G. Elba, Wadi El Kileibitat, $7 \mathrm{~km}$ N Wadi Shelatin, frontier station, 10.2.1967; Osborn and Helmy s.n. (CAI)-Wadi Aideib, sandy soil, 28.2.1938; Shabetai z 5515 (CAIM)-Exp. to G. Elba district: Wadi Aideib, 20.1.1962; V. Täckholm et al. s.n. (CAI)-Exp. to G. Elba district; Wadi Yahameib, 22.1.1962; V. Täckholm et al. s.n. (CAI)-Wadi Yahameib, 4.11.2005; Ali s.n. (CAI)-Wadi Kansisrob, 3.2.1962; V. Täckbolm et al. s.n. (CAI)-G. Elba: Wadi Kansisrob, 1.2.1979; Boulos 12878 (CAIRC)-G. Elba: G. Alafoot, 7.2.1962; V. Täckholm et al. s.n. (CAI)-Wadi Bir Akwamtra, rocky slope, 19.2.1967; Osborn and Helmy s.n. (CAI)-Wadi Rabdeît, on rock, 21.1.1933; Shabetai z 2335 (CAIM)-Wadi Rabdeît, on rock, 29.1.1933; Shabetai z 2335 (CAIM)-Bot. Dept. Exc. to G. Elba , 23.1-27.1.1929; G. Täckholm s.n. (CAI)-G. Elba, 18.1.1930; Hassib s.n. (CAI)-G. Elba, 1.1929; G. Täckholm s.n. (CAI)-Wadi Mawaw, 28.1.1962; V. Täckholm et al. s.n. (CAI)-Wadi El Shallal, 24.1.1962; V. Täckholm et al. s.n. (CAI)-Gorge across G. El Shallal, 24.1.1962; V. Täckholm et al. s.n. (CAI)-Exp. to G. Elba district: Wadi Oolak, 27.1.1962; V. Täckholm et al. s.n. (CAI)-Exp. to G. Elba district: G. Mog, 7.2.1962; V. Täckholm et al. s.n. (CAI).

Habitat: Red Sea sandy plains; gravelly hillsides and rocky slopes in adjacent wadies. Flowering: January-February.

Distribution: Egypt: Gebel Elba district (SE corner of Egypt): inner plateau of the Arabian Desert, Red Sea mountains and coastal plains (Fig. 4). It occurs in N Africa (SE Egypt), NE Tropical Africa (Sudan) and Arabian Peninsula (W Saudi Arabia, S Yemen, Dhofar) in temperate Asia.

Note: Pancratium tortuosum is closely allied to $P$. trianthum. However, the 2-valved spathe, short $(5-8 \mathrm{~mm})$ corona teeth, short $(6-10 \mathrm{~mm})$ free part of filaments and the base of leaves (truncate, with scarious-membranous sheath) are diagnostic characters of $P$. tortuosum.

\section{Pancratium trianthum Herb., new to Egypt}

Pancratium trianthum Herb., Ann. Nat. Hist., ser. 1, 4: 28 (1840); Maire, Fl. Afr. Nord 6: 39 (1959); Morton, Kew Bull. 19 (2): 340 (1965); Hepper, Fl. W. Trop. Afr., ed. 2, 3(1): 136 (1968); I. Björnstad, Norw. J. Bot. 20 (4): 285 (1973); D. Heller and Heyn, Consp. Fl. Orient. 6: 53 (1991) [non Baker, 1898; non Blatt., 1936; non Schwartz, 1939].

Type specimen: Senegambia, 1837; Heudelot 542 (K, holotype !-P, isotype).

Glabrous herbaceous perennial, 40-60 cm high; bulb solitary or gregarious, globose or subglobose to ovoid, 3-6 cm long, 2.5-5 cm diameter; neck (8-) $10-13 \mathrm{~cm}$ long; tunic 
30
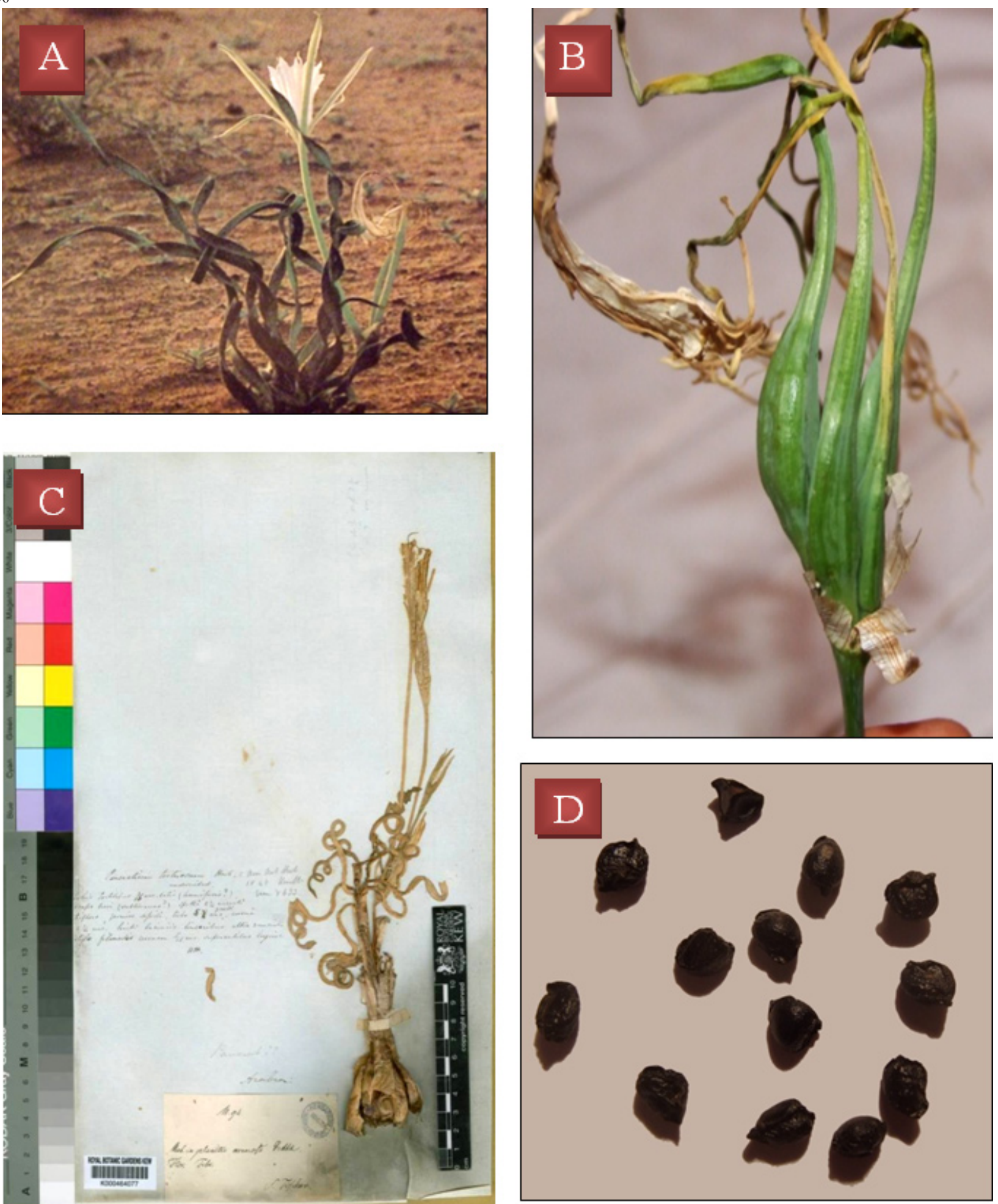

Fig. 3. Pancratium tortuosum Herb.; A: habit; B: gynoecium; C: syntype of Fischer 94 (K !); D: seeds. [Wadi Yahameib, 4.11.2005; Ali s.n. (CAI)]

dark or blackish-brown. Leaves (3-)5-10(-15), developing with or after the flowers, glaucous, linear to lanceolate-linear, (6.5) $10-30 \mathrm{~cm}$ long, $0.3-1.0 \mathrm{~cm}$ diameter, broadest at middle or slightly below, erect, slightly twisted, gradually tapering above into an acute apex, glabrous near the base; base long attenuate, without scarious-membranous sheath.
Aerial scape solitary, solid, compressed, glaucous, (6-)10$25 \mathrm{~cm}$ long, dilated at the glabrous base $(0.2-0.6 \mathrm{~cm}$ diameter). Spathe 1-valved, lanceolate or ovate-lanceolate, and with \pm contorted margins at the base, tapering above into an acute apex. Flowers (1-) 2-5, large-sized, (18-)20-24 cm long, 4-6 times as long as the spathe, fragrant, subsessile or 


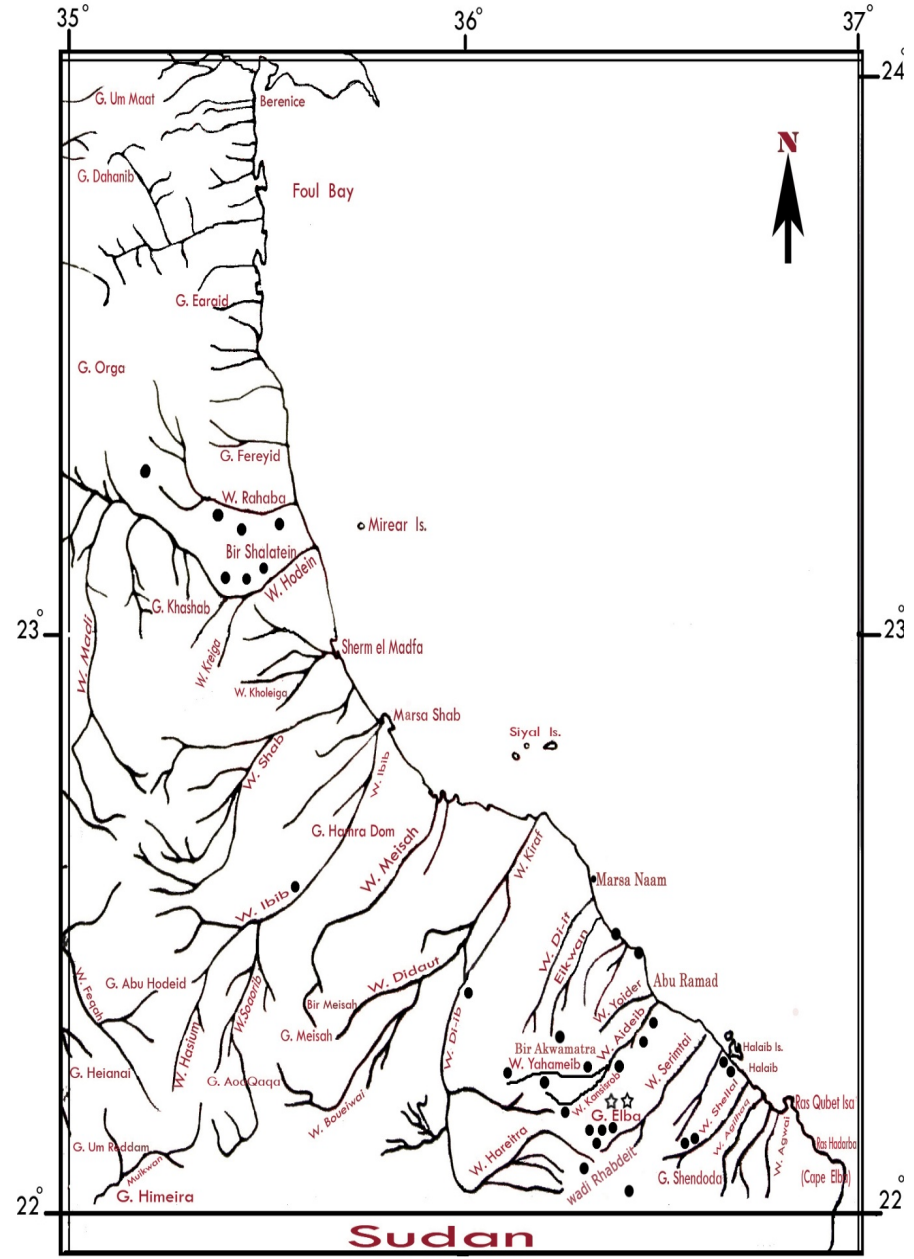

Fig. 4. Distribution map of Pancratium tortuosum $(\bullet)$ and Pancratium trianthum (מ); $\bullet$, מ specimens seen by the author

shortly-pedicelled; pedicel $0.2-0.8(-1.0) \mathrm{cm}$, shorter than the ovary; perianth white, $17-22 \mathrm{~cm}$ long; perianth-tube glaucous, $11-16 \mathrm{~cm}$ long, 2-3 times as long as the perianthsegments, white, with a narrow green mid-stripe outside, patent, 5-7 cm long, outer slightly longer and broader, narrowly spathulate-oblong to lanceolate-linear, gradually tapering above to a mucronate apex, inner linear-lanceolate, acute or mucronulate; perianth-segments 1.5-2 times as long as the corona; corona (2.5-)3-4 x 2-3 cm, margin 12-teethed; teeth triangular-acuminate (subulate), 7-10 $\mathrm{mm}$ long, shorter than the free filaments; free part of filament glabrous, 10-15(-20) mm long; anthers yellow, 6-10 $\mathrm{mm}$ long, 1.0-1.5 mm diameter; ovary glaucous, glabrous, narrowly ellipsoid, $2-3 \mathrm{~cm}$ long, $0.5-1.0 \mathrm{~cm}$ diam.; style glaucous, filiform, (18-)20-22 cm long; stigma glaucous, obscurely 3-lobed, slightly shorter than the perianth-segments (about $0.7-1.0 \mathrm{~cm}$ shorter). Capsule ellipsoidal or cylindrical, c. $3 \times 2 \mathrm{~cm}$ (from type spec.); seeds not seen (Fig. 5).

Representative specimens examined: Gebel Elba: Botany Department Excursion to Gebel Elba district, 23-27.10.1929; Hefnawy and G. Täckholm s.n. (CAI)-
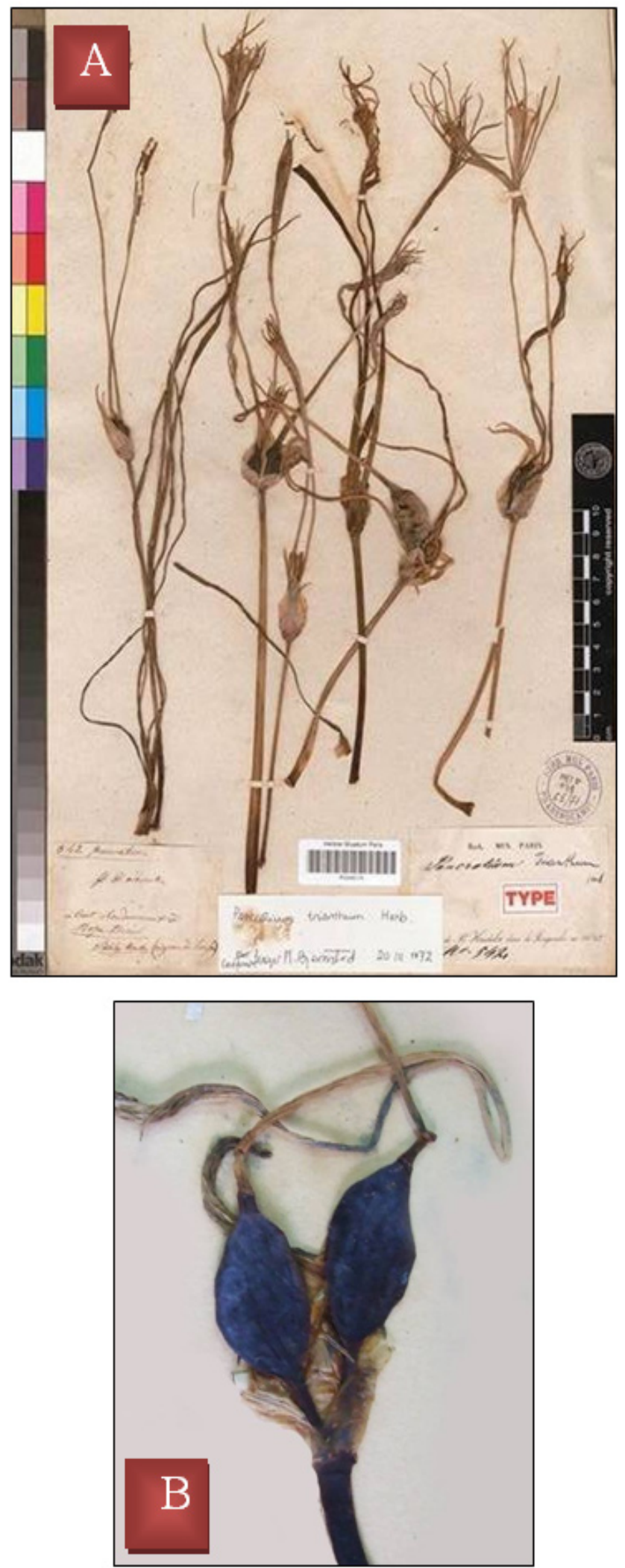

Fig. 5. Pancratium trianthum Herb. A: Type specimen: Heudelot 542 (K, holotype !). B: Gebel Elba district, 14-16.10.1933: Fahmy and Hassib s.n. (CAI). 1-valved, bifid spathe 
32

Botany Department Excursion to Gebel Elba district, 1416.10.1933; Fahmy and Hassib s.n. (CAI).

Flowering: October-November.

Distribution: Gebel Elba region in Egypt (Fig. 4). It occurs in North Africa (Morocco, Algeria, Tunisia, Egypt, Sahara), Tropical Africa (W, W-C, NE) eastwards to Arabian Peninsula (Saudi Arabia).

Note: In Egypt, our herbarium specimens are in flower, and the capsule is not seen. Therefore, the size of the capsule is obtained from the type specimen (Heudelot 542). Pancratium trianthum is closely allied to $P$. tenuifolium Hochst. ex A. Rich. and P. maximum Forssk. The three species are characterized by the presence of 1 -valved spathe, narrow leaves ( $1 \mathrm{~cm}$ or less), and glaucous or green style and stigma. Pancratium trianthum is easily distinguished from $P$. tenuifolium by having glabrous leaves and scape (v.s. finely pubescent); (1-) 2-5-flowered inflorescences (v.s. 1(-2); shorter (5-7 cm long) perianth-segments (v.s. longer, $7-10 \mathrm{~cm}$ long); and contorted margins of the spathe (v.s. not convolute). On the other hand, $P$. trianthum can be distinguished from $P$. maximum by its glaucous leaves (v.s. green); (1-)2-5-flowered inflorescences (v.s. solitary flower); shorter $(5-7 \mathrm{~cm}$ long) perianth-segments (v.s. longer, $8-10 \mathrm{~cm}$ long) and shorter (2.5-4 cm long) corona (v.s. longer, $4-5 \mathrm{~cm}$ long). Moreover, $P$. maximum is endemic to SW Arabia.

4. Pancratium sickenbergeri Asch. and Schweinf. ex Barb.-Boiss. and Barbey, Herb. Levant 158 (1882); Boiss., Fl. Orient. 5: 153 (1882); Asch. and Schweinf., GatenZeit. (Wittmack) 2: 345 (1883)-Ill. Fl. Égypte, Mém. Inst. Égypt. 2:150 no. 1032 (1887); Asch. in Asch. and Scweinf., Ibid., Suppl.: 805 no. 243(1889); Baker, Handb. Amaryll. 118 (1888); Post, Fl. Syr., Pal. et Sn., ed. 1, 777 (1896); Sickenb., Contrib. Fl. Ếgypte, Mém. Inst. Egypt. 4 (2): 291 (1901); Muschl., Man. Fl. Egypt 1: 234 (1912); Ramis, Tabell. Fl. Aegypt. 59 (1929); Dinsm. in Post, Fl. Syr., Pal. et Sn., ed. 2, 2: 609 (1933); Blatt., Fl. Arab. 456 (1936); Täckh. and Drar, Fl. Egypt 3: 363 (1954); Täckh., Stud. Fl. Egypt, ed. 1, 457 (1956)-Ibid., ed. 2, 657 (1974); Abdallah et al., Notes A.R.C. Herb. Egypt 6: 23 (1984); Collonette, Ill. Flow. Saudi Arabia 45 (1985); Danin et al., Willdenowia 15 (1): 308 (1985); Feinbrun in Zohary, Fl. Palaest. 4: 109 (1986); El Husseini and Hosni, Taeckholmia 12: 83 (1989); Migahid, Fl. SA, ed. 3, 3: 13 (1989); D. Heller and Heyn, Consp. Fl. Orient. 6: 53 (1991); El Hadidi and Fayed, Taeckholmia 15: 189 (1995); Collonette, Wildflow. Saudi Arabia 40 (1999); Boulos, Fl. Egypt 4: 88 (2005).

\section{Synonyms}

P. sickenbergeri Asch. and Schweinf. ex Barb.-Boiss. and Barbey var. desertorum Sickenb., Contrib. Fl. Égypte, Mém. Inst. Egypt. 4(2): 292 (1901).

Type specimen: Described from Egypt [El Arich, Tell ech Cheikh et G. Ekfehn, Grande, Forêt pétrifiée et dis- perse dans le desert a l'Est du Caire]. The illustration (fig. 1) in Asch. and Schweinf., Garten-Zeit. (Wittmack) 2: 345 (1883).

P. sickenbergeri Asch. and Schweinf. ex Barb.-Boiss. and Barbey var. littorale Sickenb., Contrib. Fl. Égypte, Mém. Inst. Egypt. 4(2): 292 (1887).

Type specimen: Described from Egypt [Tell ech Cheikh, pays d'el Arich, 1892]. The illustration (Fig. 2) in Asch. and Schweinf., Garten-Zeit. (Wittmack) 2: 345 (1883).

Glabrous herbaceous perennial, $30-40 \mathrm{~cm}$ high; bulb solitary, ovoid, 4-10 cm long, 2-5 cm diameter; neck 5-20 cm long; tunic dark or blackish-brown. Leaves 3-9(-13), developing after flowers, glaucous, linear to lanceolatelinear or lorate, (8-)10-30 cm long, 0.3-1.0 cm diameter, broadest at the middle or below, decumbent over the soil surface, spirally twisted along its whole length, gradually tapering above into acute apex, glabrous near the base; base truncate, without scarious-membranous sheath. Aerial scape solitary, solid, compressed, glaucous, $10-17 \mathrm{~cm}$ long, dilated at the tip (0.3-0.5 cm diameter), glabrous. Spathe 2-valved, unequal, imbricate, elongated ovoid or lanceolate-subulate, $3-5 \mathrm{~cm}$ long, (0.7-) 1-2 cm diameter, gradually tapering above into an acute apex. Flowers 2-5(-6), short, $7-10 \mathrm{~cm}$ long, 2-3 times as long as the spathe, fragrant, shortly-pedicelled; pedicels stout, $0.3-1.0 \mathrm{~cm}$ long, shorter than the ovary; perianth white, $6-9 \mathrm{~cm}$ long; perianth-tube 2-4 cm long, as long as the perianth-segments or commonly shorter; perianth-segments 3-5 cm long, white, with a broad green mid-stripe outside, outer rather longer and wider, narrowly spathulate, obtuse to subacute at the mucronate or shortly cuspidate apex; inner lanceolate or ovate-elliptic, acute or subacute, perianth-segments up to 1.5 times as long as the corona; corona $3-4 \times 2-3 \mathrm{~cm}$, margin 12-teethed; teeth narrowly attenuate-triangular, 4-6 $\mathrm{mm}$ long, 2-3 $\mathrm{mm}$ diameter, longer than the free filament; free part of filament glabrous, 3-5 mm long; anthers yellow, 5-10 mm long, 1.0-1.5 mm diameter; ovary glaucous, glabrous, ovoid or oblong-ovoid, $1-2 \mathrm{~cm}$ long, $0.5-1.0 \mathrm{~cm}$ diameter; style white, filiform, $6-8 \mathrm{~cm}$ long; stigma white, obscurely 3-lobed, shorter than the perianth-segments (about $0.5-0.7 \mathrm{~cm}$ shorter). Capsule broadly ellipsoid or ovoid, $1.5-2.5 \mathrm{~cm}$ long, $1.5-2.0 \mathrm{~cm}$ diameter; seeds irregularly angular and compressed or cone-shaped, 6-9 mm long, 3-7 mm diameter; testa black, sublastrous, minutely and closely foveolate (Fig. 6).

Representative specimens examined: Sinaitic sector of the Mediterranean coast: N Sinai:El Arish, 20.3.1928; G. Täckholm z 1955 (CAI, CAIM)-N Sinai: 70 km W El Arish, near Misfak, sabkha (salt marsh), 23.2.1984; Gibali 26 (CAIRC)-N Sinai: Bir El Abd, 10.9.2004; Ali s.n. (CAI). Isthmic Desert, Sinai: El Midan, $20 \mathrm{~km} \mathrm{~S} \mathrm{El} \mathrm{Arish,}$ 2.2.1956; Boulos s.n. (CAI)-Sinai, $20 \mathrm{~km} \mathrm{~S} \mathrm{El} \mathrm{Arish,} \mathrm{15.6.}$ 

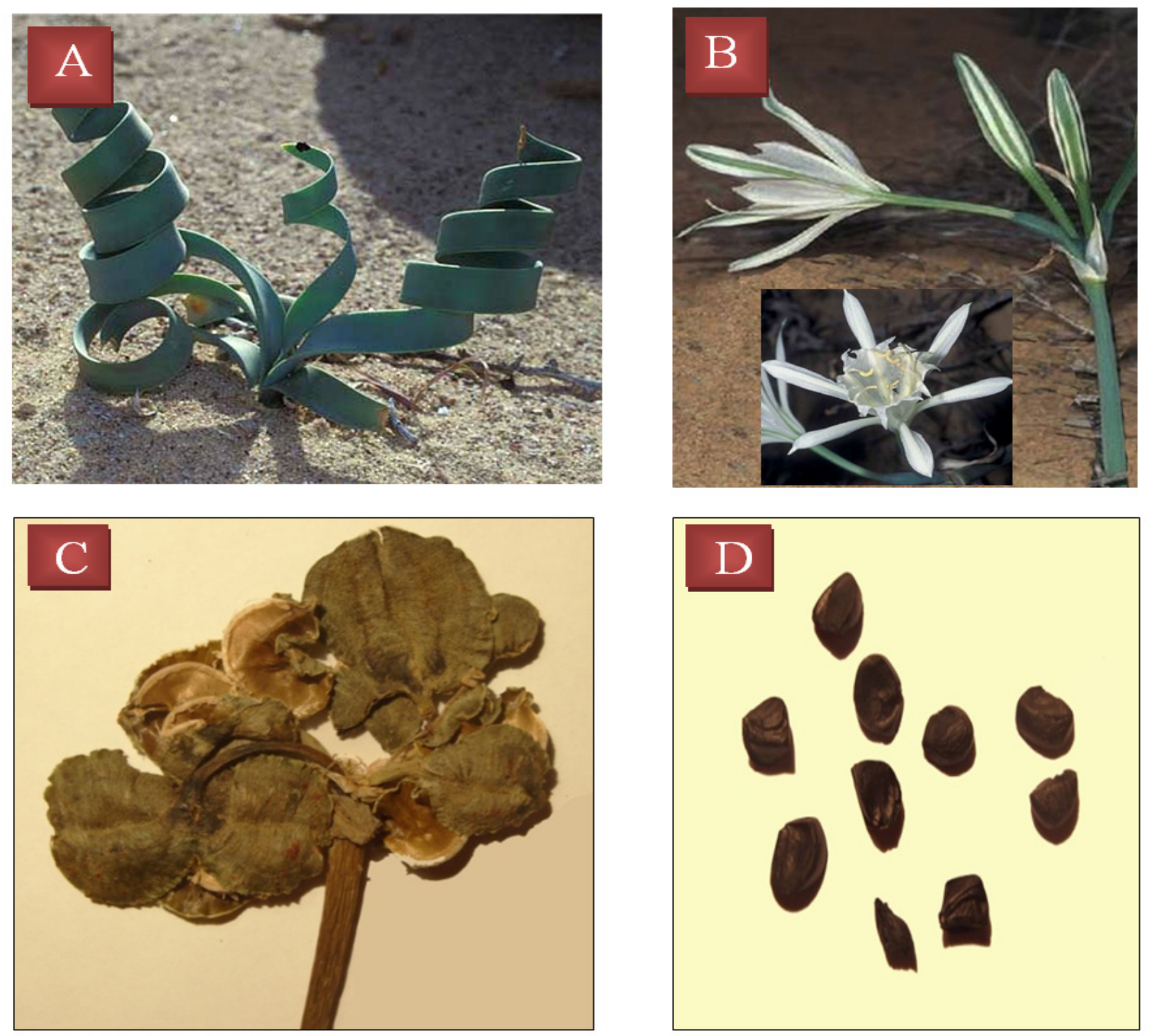

Fig. 6. Pancratium sickenbergeri Asch. \& Schweinf. ex Barb.-Boiss. and Barbey; A: habit; B: inflorescence (side, front view); C: mature capsules; D: seeds. [N Sinai: Bir El Abd, 10.9.2004; Ali s.n. (CAI)]

1960; Diab s.n. (CAI)-Wadi El Arish, in sand, 10.4.1929; Shabetai z 810 (CAIM)-Wadi El Arish, 18.3.1955; Boulos s.n. (CAI)-Sinai: Rawafaa, 19.9.1965; Eid 37 (CAIM)Wadi el Tih, 20.2.1930; Oliver s.n. (CAI). Galala Desert, Tell el kebir, in a wadi on the hill side, 23.2.1923; Simpson 1817 (CAIM)-Inshas Desert, 9.1.1953; El Hadidi s.n. (CAI)-Wadi el Fûl, to the left of Cairo-Suez Road, 14.10.1960; V. Täckholm et al. s.n. (CAI)-Cairo-Suez Road, at kilo 20, 10.3.1930; Oliver s.n. (CAI)-Cairo-Suez Road, at kilo 28, 17.10.1957; Ibrabim s.n. (CAI)-CairoSuez Road, at kilo 32, 22.12.1951; Ayyad s.n. (CAI)-CairoSuez Road, at kilo 32, 22.12. 1951; Kamel s.n. (CAI)-Suez desert Road, at kilo 35, Spring 1957; Imam s.n. (CAI)-Suez road, 2.3.1956; Montasir s.n. (CAI)-Cairo-Suez Road, 3.1.1962; V. Täckholm et al. s.n. (CAI)-Wadi Anqâbiya, 24 km E Cairo-Suez Road, 1.2.1945; Davis and Shabetai z 6294 (CAIM)-Wadi Anqâbiya, 4.1950; Ibrahim s.n.
(CAI)-Wadi Anqâbiya, 28.1.1956; Imam s.n. (CAI)-Wadi Anqâbiya near the Suez Road, 16.3.1956; El Batanouny s.n. (CAI)-Wadi Liblab, G. el Ahmar, 17.3.1969; Wissa and Nabih 3035 (CAIM)-G. El Khashab, on a calcareous hill, $25 \mathrm{~km}$ E El Ma’adi, 22.12.1931; Shabetai z3002 (CAIM)-G. Yahmûm el Asmar area, Cairo-Suez Road, 8.11.1960; V. Täckholm et al. s.n. (CAI)-Great Petrified Forest, red sand, 13.10.1922; Simpson 1668 (CAIM)-Wadi Digla, 25 km E El Ma’adi, 16.3.1969; Abdallah, Costantin and Khattab s.n. (CAIM)-G. Yahmûm el Abraq, $18 \mathrm{~km} \mathrm{E}$ Suez Road, 5.3.1945; Shabetai 6542 (CAIM)-El Saff Desert: Wadi el Hay, 21.3.1960; V. Täckholm et al. s.n. (CAI). Red Sea coast: Sinai: El Shatt, sandy plain, 12.4.1937; Shabetai z 3961 (CAIM).

Habitat: Sandy desert wadies, waste ground and roadsides, sandy or calcareous hill slopes, gravely plains, sometimes in red or saline soil. Flowering: September-October. 


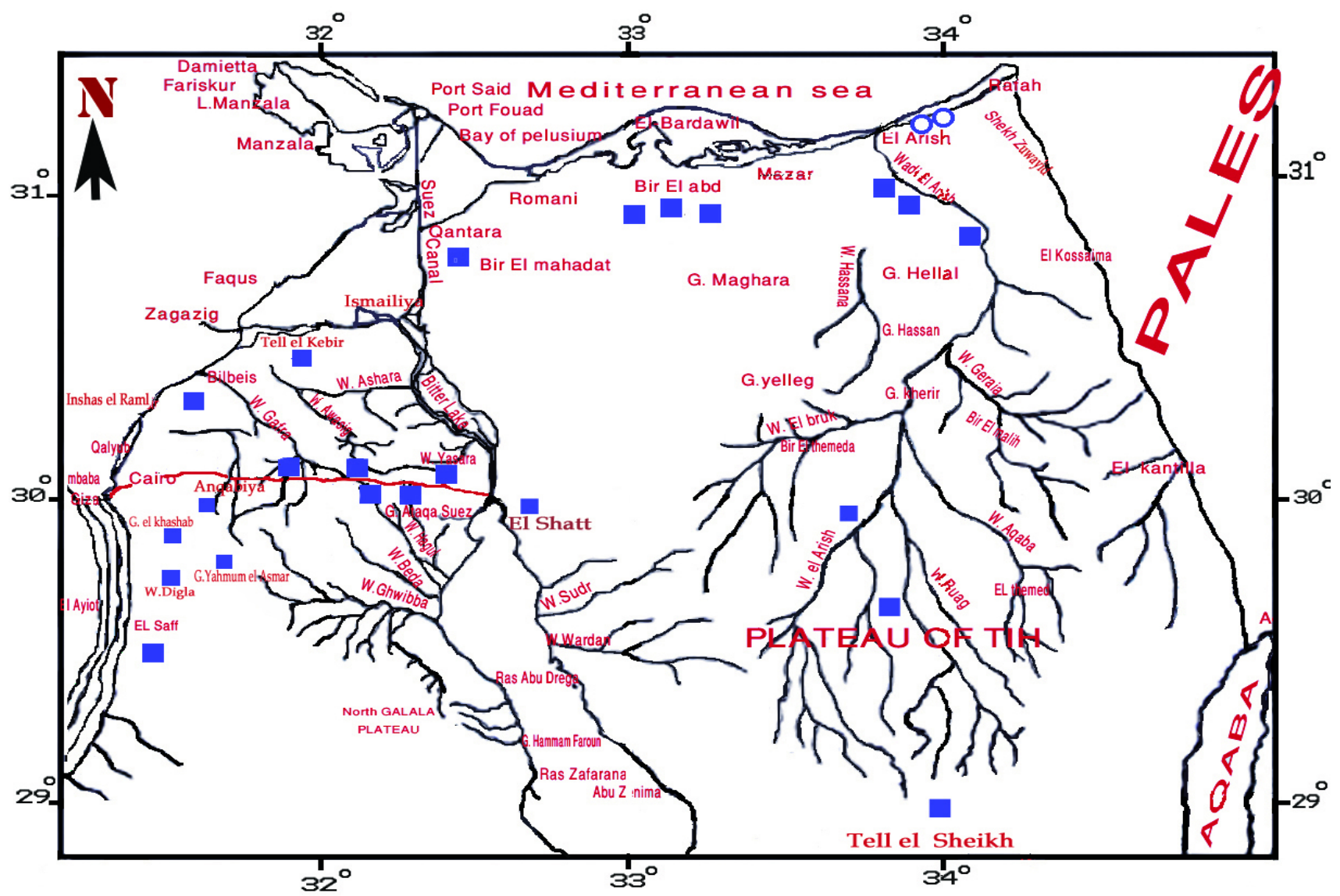

Fig. 7. Distribution map of Pancratium sickenbergeri; $\square$ specimens seen by the author; $O$ literature record for P. maritimum

Distibution: Egypt: Along the Sinaitic sector of the Mediterranean coastal land (Fig. 7) and along the Gulf of Suez and in the Eastern desert (N Galala). It occurs in N Africa (Egypt), W Asia (Palestine, Sinai) and Arabian Peninsula (Saudi-Arabia).

5. Pancratium arabicum Sickenb., Contrib. Fl. Egypte, Mém. Inst. Egypt. 4: 290 (1901); Muschl., Man. Fl. Egypt 1: 233 (1912); Ramis, Tabell. Fl. Aegypt.: 59 (1929); Dinsm. in Post, Fl. Syr., Pal. et Sn., ed. 2, 2: 609 (1933); Blatt., Fl. Arab. 456 (1936); Täckh. and Drar, Fl. Egypt 3: 361 (1954); Täckh., Stud. Fl. Egypt, ed. 1, 457 (1956)Ibid., ed. 2, 657 (1974); El-Husseini and Hosni, Taeckholmia 12: 83 (1989); D. Heller and Heyn, Consp. Fl. Orient. 6: 53 (1991); El Hadidi and Fayed, Taeckholmia 15: 189 (1995); El Hadidi, Fl. Aegyptiaca 1(1): 148 (2000); Boulos, Fl. Egypt 4: 87 (2005).

Type specimen: Cultivated specimen at Kew from bulbs, received by Sickenberger from Egypt (at Mer near Alexandria), 4.1894 (K, syntype !).

\section{Synonyms}

P. aegyptiacum M. Roem., Fam. nat. syn. monogr. 4: 180 (1847); Sickenb., Contrib. Fl. Egypte, Mém. Inst. Egypte 4: 292 (1901); Muschl., Man. Fl. Egypt 1: 234 (1912); Ramis, Tabell. Fl. Aegypt. 59 (1929).
Glabrous herbaceous perennial, $60-90 \mathrm{~cm}$ high; bulb solitary or gregarious, usually deeply buried, globose or subglobose to elongated ovoid, $7-12 \mathrm{~cm}$ long, $5-10 \mathrm{~cm}$ diameter, narrowed above into a long cylindrical neck; neck 10-40(-60) cm long; tunic dark or blackish-brown. Leaves 5-10(-13), developing after the flowers, glaucous, broadly linear to oblong-lanceolate or lorate, $30-80 \mathrm{~cm}$ long, 1-2-3 $\mathrm{cm}$ diameter, broadest at middle or slightly above, erect, straight to arched or often twisted near the apex, abruptly narrowed above into obtuse to subacute apex, glabrous near the base; base truncate, with long membranous sheath. Aerial scape solitary, solid, compressed, glaucous, 17-24 $\mathrm{cm}$ long, dilated at the tip (1.0-3.0 cm diameter), glabrous. Spathe 2-valved, unequal, imbricate, lanceolate or ovatelanceolate, (5-) 6-9 cm long, 2-3 cm diameter, gradually or abrubtly tapering above into a long acuminate apex. Flowers 5-14, medium-sized, 13-18 cm long, 1.5-2 times as long as the spathe, fragrant, pedicelled; pedicel stout, 1-2 $\mathrm{cm}$ long, commonly shorter than the ovary or rarely equal, perianth white, $11-16 \mathrm{~cm}$ long; perianth-tube (7-)8-12 $\mathrm{cm}$ long, 1.5-2 times as long as the perianth-segments; perianth-segments white, with a broad green mid-stripe outside (4-6 mm diameter), patent, 5-7 cm long, 5-10 mm diameter; outer narrowly spathulate to lanceolate-oblong or linear-oblong, obtuse to subacute at the mucronate or shortly cuspidate apex; inner slightly shorter and narrower, lanceolate or oblong-lanceolate, acute or subacute, 

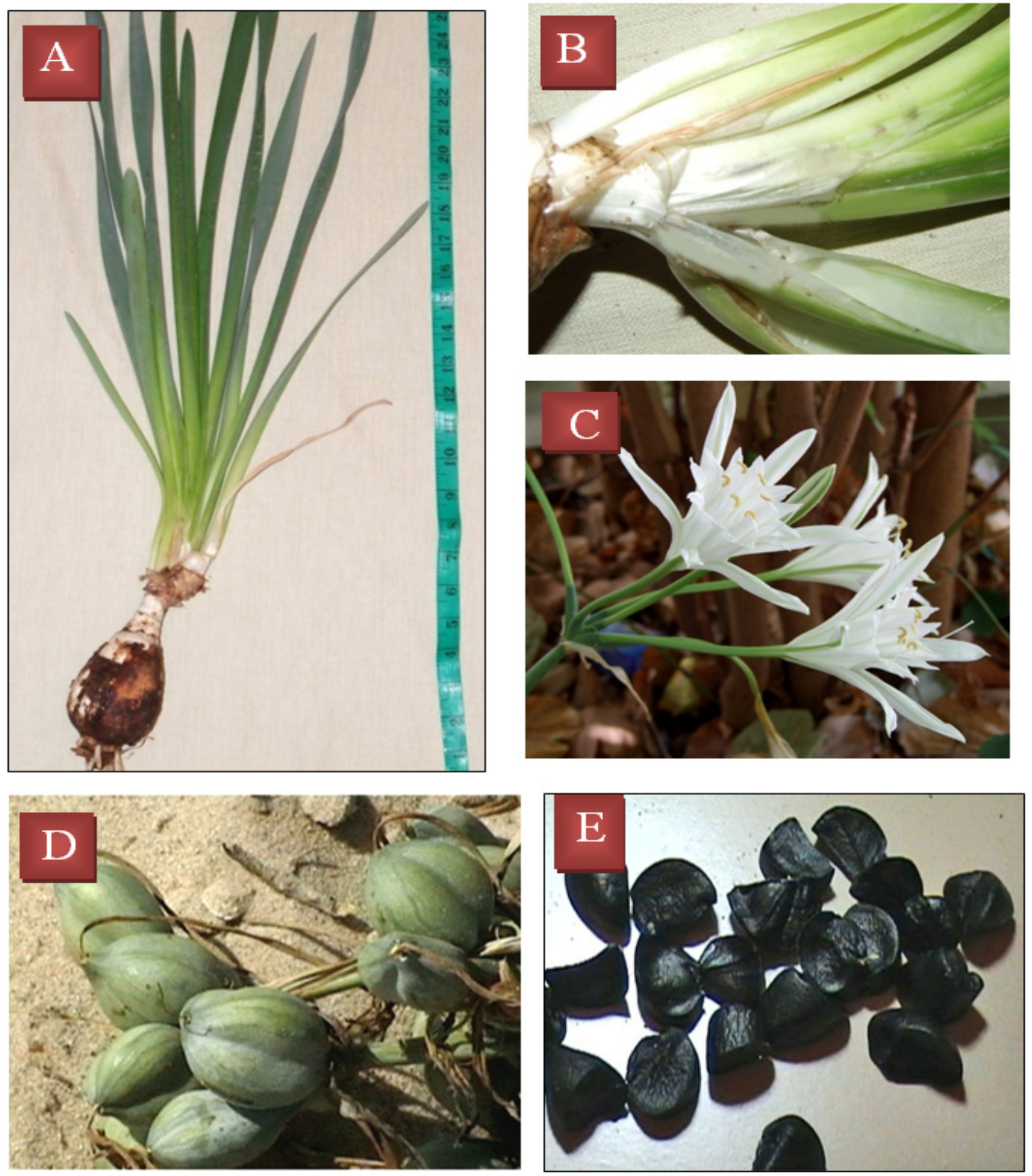

Fig. 8. Pancratium arabicum Sickenb. A: habit; B: scarious sheath at leaf-base; C: inflorescence; D: the infructescence; E: seeds. [N Sinai: Rafah-Sheikh Zuwayid, 23.9.2006; Ali s.n. (CAI)]

1.5-2 times as long as the corona; corona $2.5-4.0 \times 2-4 \mathrm{~cm}$, margin 12-teethed; teeth attenuate-triangular, $5-8 \mathrm{~mm}$ long, acute, as long as the free filament or shorter; free part of filament glabrous, 6-10 mm long; anthers yellow, 10-17 $\mathrm{mm}$ long, $1.0-1.5 \mathrm{~mm}$ diameter; ovary glaucous, glabrous, ovoid-oblong to narrowly ellipsoid, $2-3 \mathrm{~cm}$ long, $0.5-1.0$ cm diameter; style white, filiform, $10-16 \mathrm{~cm}$ long; stigma white, obscurely 3-lobed, as long as the perianth-segments or commonly shorter (about $0.5-1.0 \mathrm{~cm}$ shorter). Capsule oblong-ovoid or broadly ellipsoid to broadly cylindrical, (2.5-)3-4 (-5) cm long, (2-)2.5-3.5 cm diameter, commonly with a peg-like projection; seeds irregularly angular and 


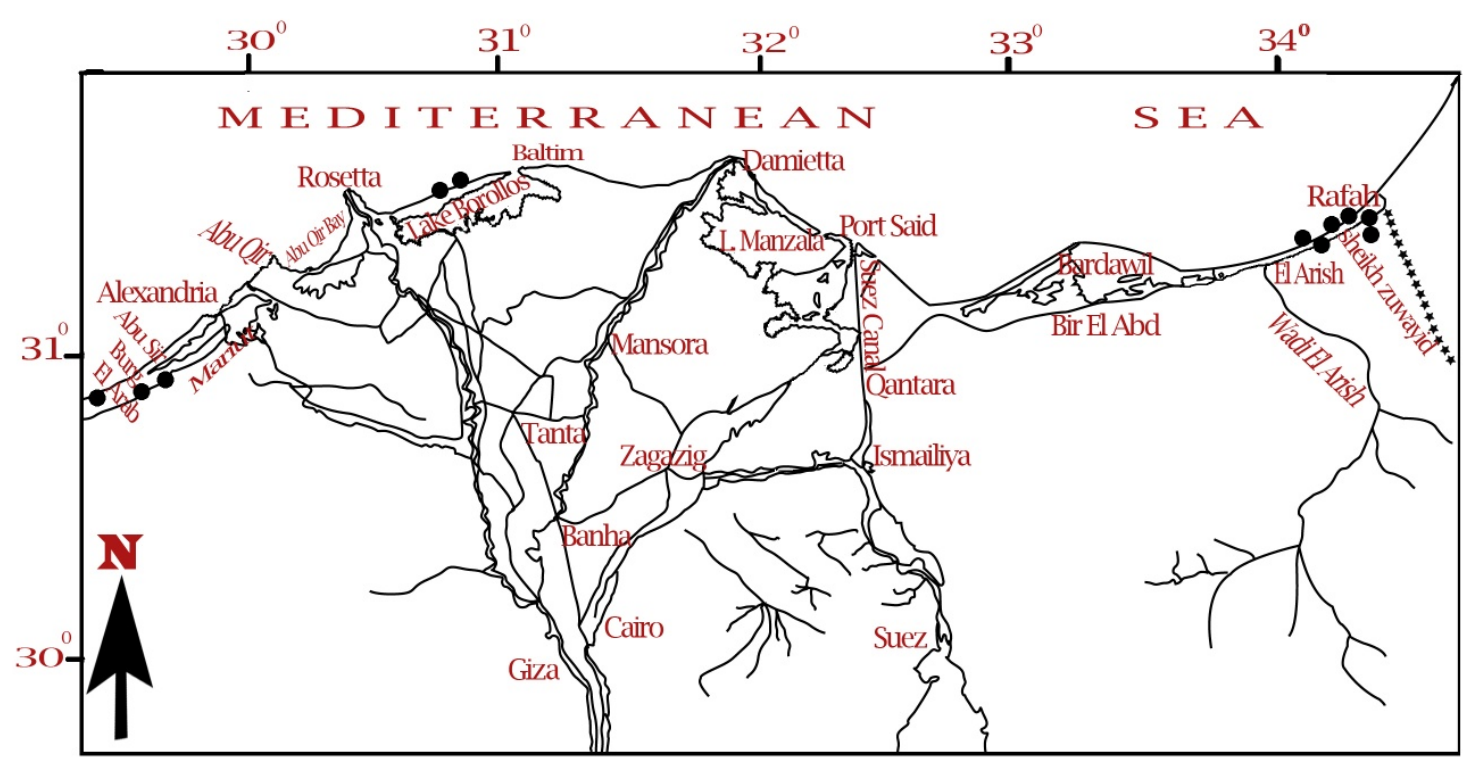

Fig. 9. Distribution map of Pancratium aegyptiacum; • specimens seen by the author

compressed or cone-shaped, 10-14 mm long, 8-10 mm diameter; testa black, sublustrous, minutely and closely foveolate (Fig. 8).

Representative specimens examined: Western Mediterranean coast: W Burg El Borollos, in the sandy dunes, 16.8.1961; V. Täckholm, Kassas and El Hadidi s.n. (CAI)Emad, $3 \mathrm{~km} \mathrm{~W}$ Burg El Borollos, in sand, 16.8.1961; $V$. Täckholm, Kassas and El Hadidi s.n. (CAI)-Burg El Borollos, 9.1952; Kassas s.n. (CAI)-Burg El Borollos, 17.8.1961; V. Täckholm and El Hadidi s.n. (CAI)-MariutBurg El Arab, 15.3.1928; G. Täckholm s.n. (CAI). Siniatic sector of the Mediterranean coast: Sinai: Rafah, near the police station, 23.3.1928; G. Täckholm s.n. (CAI)-N Sinai: Rafah, sandy dunes, 15.8-8.9.1951; V. Täckholm, Kassas and Jackos s.n. (CAI)-N Sinai: Rafah, in maritime sands, 4.3.1987; Boulos 786 (CAI)-N Sinai: Rafah, near the beach, 4.3.1987; Gibali 786 (CAIRC)-N Sinai: Rafah-Sheikh Zuwayid, 23.9.2003; Ali s.n. (CAI)-N Sinai: Rafah, Bir El Meleha, at the coast, 23.3.1928; G. Täckholm s.n. (CAI).

Habitat: Sandy maritime seashores and sand dunes. Flowering: July-September.

Distibution: Endemic to Egypt and occurs all along the Mediterranean coastal region and Sinai (Fig. 9). It occurs in N Africa (Egypt) and Sinai.

Notes: Several authors such as: Sickenberger (1901), Muschler (1912), Ramis (1929) treated P. aegyptiacum as a distinct species, while Täckholm and Drar (1954), Täckholm (1974) treated it as a synonym to P. maritimum. Pancratium arabicum is misidentified as P. maritimum. Therefore, we have examined at some length the diagnostic characters of $P$. arabicum from fresh material during intensive field trips and from different Egyptian herbaria. We concluded that in the absence of leaves, longer (11-16 $\mathrm{cm})$ perianth with longer $(8-12 \mathrm{~cm})$ perianth-tube, longer $(5-7 \mathrm{~cm})$ perianth-segments with broader $(4-6 \mathrm{~mm})$ green mid-stripe and the size of the capsule $(3-5 \times 2.5-3.5 \mathrm{~cm})$ distinguish $P$. arabicum.

Although Täckholm and Drar (1954, p. 359) cited $P$. aegyptiacum as a synonym to $P$. maritimum, they again cited Sickenberger specimens (recorded from El Arish; "Tell el Sheikh" and identified as P. aegyptiacum) as well as those of Schweinfurth (recorded from Cairo; Birket el Sab' and Damietta and identified as $P$. aegyptiacum) with query as P. arabicum (1954, p. 361). However, the diagnostic characters of $P$. aegyptiacum emended by Sickenberger are: broad leaves, long perianth-tube $(10-12 \mathrm{~cm})$, long pedicel at anthesis $(1.0-1.5 \mathrm{~cm})$ and oblong-ovoid capsule valves are scattered throughout the range of variation in $P$. arabicum without strong geographical bias.

\section{References}

Andrews FW (1956). The Flowering Plants of the Sudan. Vol. 3. Arbroath, Scotland.

Ascherson P, Scweinfurth, G (1883). Pancratium sickenbergeri Aschs. et Schwf. (Amaryllidaceae) subtr. 3. Cyathiferae Bent. et Hook.). Gartenzeitung (Wittmack) 2:345-348.

Ascherson P, Scweinfurth, G (1887). Illustration de la Flore d'Egypte. Mém Inst Égypt 2(1):23-260.

Ascherson P, Scweinfurth, G (1889). Supplément a l'Illustration de la Flore d'Egypte. Mém Inst Égypt 2(1):743-821.

Ascherson P (1889). Florula Rhinocoluraea, p. 786-810. In: Ascherson P and Scweinfurth G (Eds.). Supplément.

Ascherson P (1889). Florula Sirbonis, p. 810-814. In: Ascherson P, Scweinfurth G (Eds.). Supplément.

Baker JG (1888). Handbook of the Amaryllideae including the Alstroemerieae and Agaveae. George Bell and Sons, London.

Baker JG (1898). Pancratium L., p. 406-407. In: ThisteltonDyer WT (Ed.), Flora of Tropical Africa. Vol. 7. L. Reeve 
and Co. Ltd., Ashford, Kent.

Barbey-Boissier C, Barbey-Boissier W (1882). Herborisation au Levent. G. Bridel, Lausanne.

Björnstad IN (1973). A revision of the genus Pancratium in Africa south of the Sahara. Norweg J Bot 20(4):281-291.

Blatter E (1916). Flora of Aden. Rec Bot Surv India 7(3):337418.

Blatter E (1936). Flora Arabica. Rec Bot Surv India 8(5):451501.

Boissier E (1854). Diagnosis Plantarum Orientalium Novarum, ser. 1, Fasc. 13:18. Leipzig and Paris.

Boissier E (1882-1884). Flora Orientalis. Vol. 5 (1, 2). George $\mathrm{H}$ Genève, Basel and Lyon.

Boulos L (2005). Flora of Egypt (Alismataceae-Orchidaceae). Vol. 4. Al Hadara Publishing, Cairo, Egypt.

Brummitt RK, Powel CE (1992). Authors of Plant Names. Royal Botanic Gardens, Kew.

Collenette S (1985). An illustrated Guide to the Flowers of Saudi Arabia. Scorpion Publishing Ltd. London.

Collenette S (1991). Wildflowers of Saudi Arabia (NCWCD). East Angraving Co. Ltd., Norwich, U.K.

Dahlgren RMT, Clifford HT, Yeo P (1985). The Families of the Monocotyledons: Structure, Evolution and Taxonomy. Springer Verlag, Berlin.

Danin AM, Shmida A, Liston A (1985). Contributions to the Flora of Sinai. III. Chechlist of the species collected and recorded by the Jerusalem team 1967-1982. Willdenowia $15(1): 255-322$.

Delile AR (1813-1814). Description de l'Égypte (Tom. sec.) 3 (Florae Aegypticae Illustratio). Paris.

Dinsmore JE (1933). Pancratium L., p. 209-210. In: Post GE (Ed.) Flora of Syria, Palestine and Sinai. $2^{\text {nd }}$ Ed., vol. 2. American Press, Beirut.

Dinter K (1909). Deutsch-Südwestafrika, Flora, Forst-und landwirtschaftlige Fragmente. Leipzig.

El-Gadi A (1978). Amaryllidaceae, p. 1-5. In: Jafri SMH and ElGadi A (Eds.). Flora of Libya. Vol. 51. Al Faateh University: Flora Committee, Tripoli.

El Hadidi MN, Fayed AA (1994/1995). Materials for Excursion Flora of Egypt. Taeckholmia 15:1-233.

El Hadidi MN (2000). Geomorphology, Climate, and Phytogeographic Affinities, p. 1-25. In: El-Hadidi, MN (Ed.). Flora Aegyptiaca. vol. 1(1). The Palm Press, Cairo and Cairo University Herbarium, Giza, Egypt.

El Husseiny N, Hosni, HA (1989). Annotated list of the Flora of Sinai (Egypt). 9. Angiospermae: Hydrocharitaceae-Juncaceae. Taeckholmia 12:79-85.

Fay MF, Chase MW (1996). Resurrection of Themidaceae for the Brodiaea alliance, and recircumscription of Alliaceae, Amaryllidaceae and Agapanthoideae. Taxon 45:441-451.

Feinbrun-Dothan N (1986). In: Zohary M (Ed.). Flora Palaestina. Vol.4. Israel Academy ofSciences and Humanities,
Jerusalem.

Forsskal P (1775). Flora Aegyptiaco-Arabica. Copenhagen.

Grassi F, Cazzaniga E, Minuto L, Peccenini S, Barberis G, Basso B (2005). Evaluation of biodiversity and conservation strategies in Pancratium maritimum L. for the Northern Tyrrhenian Sea. Biodiv Conserv 14(9):2159-2169.

Heller D, Heyn CC (1991). An Annotated catalogue of the Flora of the Middle East. Conspectus Florae Orientalis. Israel Academy of Sciences and Humanities, Jerusalem, p. 6-53.

Herbert W (1837). Amaryllidaceae. J. Ridgeway and Sons, London.

Herbert W (1840). Amaryllidearum species novae. Ann Nat Hist, ser 1(4):28.

Hepper FN (1968). Amaryllidaceae, p. 131-137. In: Hepper FN (Ed.). Flora of West Tropical Africa, $2^{\text {nd }}$ Ed. Vol. 3(1). Crown Agents,London.

Hepper N, Friis I (1994). The plant of Pehr Forsskal's Flora Aegyptiaco-Arabica. Royal Botanic Gardens, Kew and Botanical Museum, Copenhagen.

Holmgren PK, Holmgren NH, Barnett LC (1990). Index Herbariorum, $8^{\text {th }}$ Ed., vol.1. New York Botanical Gaeden, New York U.S.A.

Hutchinson J (1934). Families of Flowering Plants. Vol. 2. Monocotyledons, $1^{\text {st }}$ Ed. MacMillan, London.

Hutchinson J (1959). Families of Flowering Plants, Vol. 2. Monocotyledons, $2^{\text {nd }}$ Ed. Clarendon Press, Oxford.

Jarvis C (2007). Order out of Chaos. The Linnaean Society of London and Natural History Museum, London.

Linnaeus C von (1753). Species Plantarum, $1^{\text {st }}$ Ed. Stockholm.

Linnaeus C von (1754). Genera Plantarum, $5^{\text {th }}$ Ed. Stockholm.

Mabberley DJ (1993). The Plant Book, $1^{\text {st }}$ Ed. Cambridge University Press, Cambridge.

Maire R (1959). Flore de l'Afrique du Nord. Vol. 6 Monocotyledonae. Lechevalier, Paris, p. 33-41.

Meerow AW (1995). Towards a phylogeny of Amaryllidaceae, p. 169-179. In: Rudall PJ, Cribb PJ, Cutler DF and Humphries CJ (Eds.). Monocotyledons: Systematics and Evolution. Royal Botanic Gardens, Kew.

Meerow AW, Snijman DA (1998). Amaryllidaceae, p. 83-110. In: Kubitzki K (Ed.). The Families and Genera of Vascular Plants, Vol. 3. Springer-Verlag, Berlin.

Meikle RD (1985). Flora of Cyprus, Vol. 2. Bentham-Moxon Thrust, Royal Botanic Gardens, Kew.

Mill RR (1984). Pancratium L., p. 380-381. In: Davis PH (Ed.). Flora of Turkey and the East Aegean Islands. vol. 8. Edinburgh University Press, Edinburgh.

Morison R (1680). Plantarum historiae universalis. 2(4). Oxford.

Morton JK (1965). The experimental taxonomy of the West African species of Pancratium L. (Amaryllidaceae). Kew Bull 19(2):337-347. 
38

Müller-Doblies D , Müller-Doblies U (1978). Studies on tribal systematics of Amaryllidaceae 1. The systematic position of Lapiedra Lag. Lagscalia 8(1):13-23.

Muschler R (1912). A Manual Flora of Egypt. Vol. 1. Friedlaender, Berlin.

Nordal I (1982). Pancratium L., p. 26-28. In: Polhill RM (Ed.). Flora of Tropical East Africa. (Amaryllidacea). Crown Agents, London.

Parlatore F (1854). Nuovi Generi e nuove Species di Piante monocotiledoni. Firenze.

Pax F (1887). Amaryllidaceae, p. 97-124. In: Engler A and Prantl K (Eds.). Die Natürlichen Pflanzenfamilien, $1^{\text {st }}$ Ed. vol. 2 (5). Verlag von Wilhelm Engelmann, Leipzig.

Pax F, Hoffmann K (1930). Amaryllidaceae, p. 391-430. In: Engler A and Prantl K (Eds.). Ibid., 2 ${ }^{\text {nd }}$ Ed., 15a. Verlag von Wilhelm Engelmann, Leipzig and Beriln.

Rafinesque-Schmaltz CS (1838). Flora Telluriana, vol. 4. Philadelphia.

Ramis AI (1929). Bestimmungstabellen Zur Flora Von Aegypten. Jena.

Salisbury RA (1866). The genera of plants, a fragment containing part of Liriogamae J. Van Voorst, London.
Saltz D, Ward D (2000). Responding to a three-pronged attack: desert lilies subject to herbivory by Dorcas gazelles. Plant Ecol 148:127-138.

Sickenberger G (1901). Contributions à la Flore d' Égypte. Mém Inst Égypt 4(2):167-332.

Smith GL, Garland MA (2003). Nomenclature of Hymenocallis (Amaryllidaceae) in Southeastern United States. Taxon 52(4): 805-817.

Täckholm V, Drar M (1954). Flora of Egypt. Vol. 3. Bull Fac Sci Cairo University 30:1-638.

Täckholm V (1974). Students' Flora of Egypt , $2^{\text {nd }}$ Ed. Cairo University, Beirut.

Traub HP (1936). Genera of the Amaryllidaceae. American Plant Life Society, La Jolla.

Ward D, Saltz D (1994). Foraging at different spatial scales: Dorcas gazelles foraging for lilies in the Negev desert. Ecology 75:48-58.

Wood JRI (1997). A handbook of the Yemen Flora. Aylesbury, U.K. 